\title{
Highly Balanced Gradiometer Systems Based on HTS-SQUIDs for the Use in Magnetically Unshielded Environment
}

\author{
J. Borgmann, A. P. Rijpma, H.J.M. ter Brake and H. Rogalla \\ Low Temperature Division, Faculty of Applied Physics, University of Twente, PO Box 217, 7500 AE Enschede, The Netherlands \\ P. David \\ Institut für Strahlen- und Kernphysik der Universität Bonn, D-53117 Bonn, Germany
}

\begin{abstract}
Two different concepts for gradiometer formation were tested applying high-temperature rf SQUIDs operated at $77 \mathrm{~K}$ in liquid nitrogen. All gradiometer systems are fully based on magnetometers. The first concept applies a compensating magnetometer at different positions to actively cancel the magnetic field at the location of other magnetometers. These magnetometers were arranged in an axial direction. In parts, a system of superconducting plates was used to align the relative magnetic orientation of the magnetometers. The outputs of these sensors were used to form a highly balanced electronic gradiometer. The second concept is based on electronic noise cancellation. $A$ set of three magnetometers arranged in an axial direction was used to form an electronic second-order gradiometer. Different types of reference systems based on HTS-SQUID magnetometers and fluxgate sensors were applied to the gradiometer signal for achieving a high common mode rejection of the environmental disturbances. The performance of the different systems is demonstrated in a magnetically unshielded environment as well as in a shielded environment and the common mode rejection of homogeneous magnetic fields is measured. To demonstrate the performance of the systems, biomagnetic measurements have been performed in shielded and unshielded environments.
\end{abstract}

\section{INTRODUCTION}

For many high-resolution magnetic measurements like magnetocardiology (MCG) and magnetoencephalography using SQUID sensors, high quality, environmental noise reduction techniques must be applied. In case of low- $T_{c}$ gradiometers, oppositely wire-wound pickup loops are coupled to the magnetically shielded SQUID [1]. For high-T, SQUIDs, this is currently not possible because of the lack of low-1/f-noise HTS tapes and superconducting interconnections. In the past, two different concepts have been demonstrated to form HTS gradiometric arrangements.

Firstly, planar thin-film gradiometers have been demonstrated in single as well as multilayer technology with baselines between $2 \mathrm{~mm}$ to $4.8 \mathrm{~cm}$ [2] - [4]. Secondly, electronically formed gradiometers have been used in several configurations [5] - [8]. These concepts offer the possibility for axial gradiometers with no limitation in baseline, but have several demands on the electronics for applications in unshielded environment. Furthermore, they can't be rotated in earth magnetic field because the increase of $1 / f$ noise due to flux trapping in the superconducting parts of the sensor. One solution to overcome these problems is the introduction of a compensating magnetometer in a Three SQUID Gradiometer (TSG) [9], but up to now a detailed demonstration of such a configuration has not been published.

Manuscript received September 15, 1998

This research is supported in parts by the Dutch Technology Foundation (STW) and Philips Medical Systems
For unshielded applications highly balanced systems are necessary. So far only a few methods for the improvement of the common mode rejection, all based on mechanical adjustment, suitable for single channel systems, have been presented [5], [7]. Other techniques like the use of reference SQUID magnetometers have been used to improve the common mode rejection of LTS hardware gradiometers in magnetically shielded as well as in unshielded environment [10], [11].

For the measurement of the fetal magnetocardiogram (fMCG) in an unshielded environment, well balanced, large baseline systems are needed, which can be positioned at different measurement locations [12]. Electronic gradiometers can be easily adapted for this application by combining compensation techniques with reference systems. Currently a system is under construction, which fulfills these requirements [13]. As preliminary tests for this final system, we have tested independently the compensation techniques with sensitive HTS rf-SQUIDs and the use of a three magnetometer references for the improvement of the common mode rejection of the environmental disturbances.

\section{COMPENSATION CONFIGURATION AND PERFORMANCE}

The first type of gradiometer has been used in two different configurations, applying a sensitive first-order axial electronic gradiometer with a baseline of $5 \mathrm{~cm}$ and a less sensitive compensating SQUID at two different axial positions (Fig. 1., a)). The common mode rejection of homogeneous magnetic fields for the first order gradiometers was measured to be better than 200 for the first configuration i) and more than 1500 for the second configuration ii), where superconducting plates were used for balancing [7]. For the experiments, we used in main parts HTS rf-SQUIDs (Table I), electronics, cryostat and holders, which were used

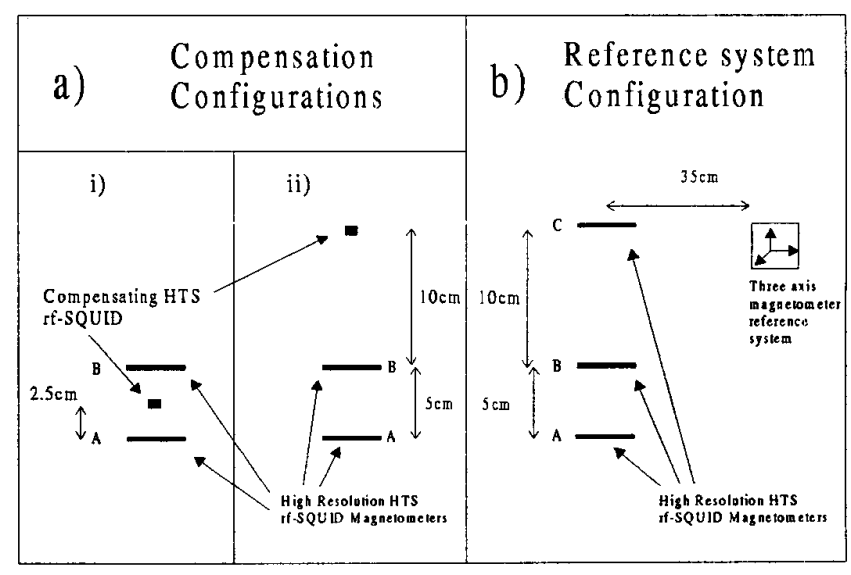

Fig.1. Schematic sketch of the two different configurations. 


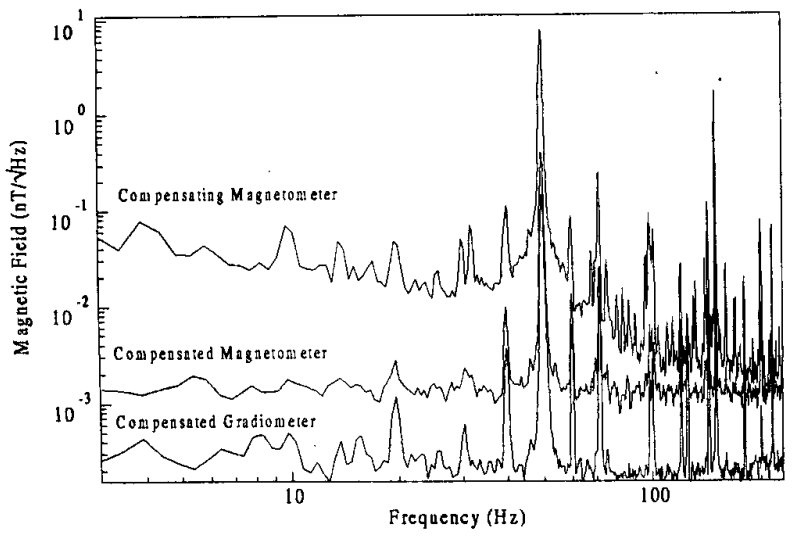

Fig.2. Simultaneously recorded spectral magnetic noise contribution of the compensating, the compensated SQUID magnetometers and electronic gradiometers in unshielded environment at research center Jülich, Germany. Due to the higher intrinsic noise of the compensating magnetometer the sensitive compensated magnetometers measure mostly the noise contribution of the compensating sensor.

and described earlier in detail [7], [14]. This HTS rf-SQUID electronics operating between 170 to $280 \mathrm{MHz}$ allowed the use of these SQUID magnetometers with high effective area reliably in all tested unshielded environments. To compensate the environmental disturbances and strong changes in background field for the sensitive magnetometers, the signal of the compensating SQUID is fed to the feedback inductance of these magnetometers via a tunable resistor. The compensation of both sensitive magnetometers by the small compensating SQUID was tuned with a homogeneous field at $1 \mathrm{~Hz}$, produced by a Helmholtz type coil set in the axial direction. The common mode rejection of this signal in the axial direction was better than $60 \mathrm{~dB}$ in both magnetometers. After this adjustment procedure the system was operated in

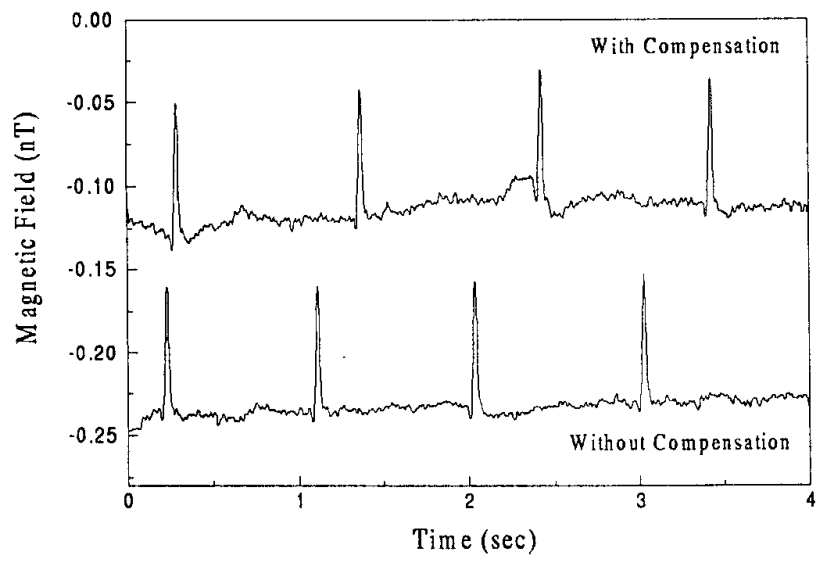

Fig.3. Magnetocardiogram recordings of an adult person measured with and without the compensation in magnetically unshielded environment at research center Jülich, Germany. The signal of the first order gradiometer was bandpass filtered between 0.1 to $47 \mathrm{~Hz}$ and notch filtered at $50 \mathrm{~Hz}$.

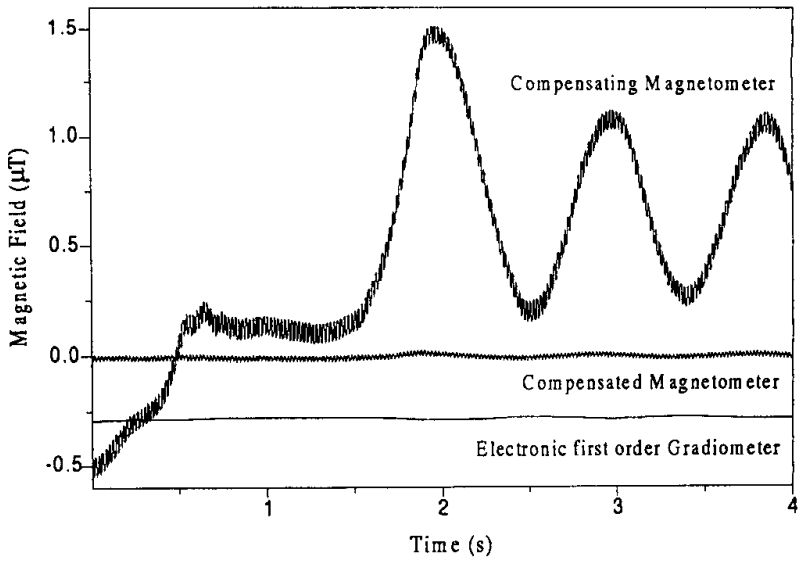

Fig.4. Comparison of different time traces simultaneously measured with the compensating magnetometer, the compensated magnetometer and the electronic gradiometer for a rotation in unshielded laboratory environment at EL/TN building, University of Twente.

an unshielded environment to measure the performance.

TABLE I

Data of the used HTS if SQUIDS

(*Thin film washer SQUIDs $8 \times 8 \mathrm{~mm}^{2}$ combined with thin film flux focussers in flip-chip technique)

\begin{tabular}{llccl} 
& $\begin{array}{l}\mathrm{L}_{\mathrm{p}} \\
{[\mathrm{pH}]}\end{array}$ & $\begin{array}{c}\text { Washer Size } \\
\text { diameter }[\mathrm{mm}]\end{array}$ & $\begin{array}{l}\mathrm{A}_{\mathrm{eff}} \\
{\left[\mathrm{mm}^{2}\right]}\end{array}$ & $\begin{array}{l}\text { White noise } \\
{[\mathrm{fT} / \mathrm{VHz}]}\end{array}$ \\
\hline SQUID A & 300 & $24 \varnothing$ & 2.91 & 94 \\
SQUID B & 300 & $24 \varnothing$ & 2.93 & 86 \\
Compensating & 150 & $3.5 \varnothing$ & 0.21 & 1300 \\
SQUID & & &
\end{tabular}

Fig. 2 shows the performance of the setup with the second compensating configuration (ii)) at research center Jülich (FZJ). At $10 \mathrm{~Hz}$, the compensated magnetometers, acting like intrinsic first order gradiometers, capable of moving in strong background fields, had a noise level typically $30 \mathrm{~dB}$ lower than the single magnetometer. This noise level represents mainly the intrinsic noise of the compensating magnetometer. At the output of the electronic gradiometer $\mathrm{A}-\mathrm{B}$, which subtracts the compensation signal from the magnetometers again, this noise is suppressed again and one achieves the first order gradiometer behavior as for a single electronic gradiometer A-B. This can also be seen in the MCG measurements at the same environment (Fig. 3).

The performance of the common mode rejection for signals caused by movements in an unshielded environment has been tested for the first compensation configuration at the EL/TN building, University of Twente. To be able to move the system in the environmental background field, the compensation should be sufficient to keep the gradiometer in the dynamic range. Figure 4 shows the behavior of the compensated magnetometer and gradiometer in comparison to the compensating magnetometer. The signal caused by the movement of the system is suppressed by $33 \mathrm{~dB}$, which is sufficient to keep the magnetometers in range. 


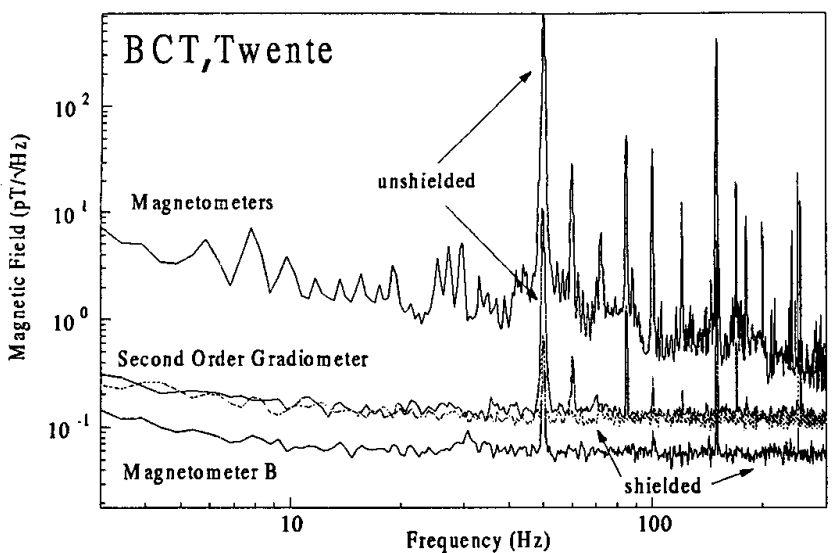

Fig.5. Simultaneously recorded spectral magnetic noise contributions of the uncompensated SQUID magnetometers and electronic gradiometers in unshielded environment with low disturbance in comparison to the signals measured inside the magnetically shielded room.

\section{REFERENCE SYSTEM CONFIGURATION AND PERFORMANCE}

For the test of the additional reference system, an axial three SQUID system was applied (Fig 1., b)) in an asymmetric baseline configuration to form an electronic second order gradiometer [11]. In general, the same type of HTS-rfSQUIDs, electronics and setup as for the compensation configuration were used, but optimized for better white noise performance of the magnetometers (Table II). The common mode rejection for homogeneous magnetic fields was again tuned in the axial direction and measured in the other orthogonal directions with a homogeneous $\mathrm{AC}$ magnetic field at $1 \mathrm{~Hz}$ generated by a large Helmholtz type coil system. For all directions, we measured a common mode rejection of better than 200 . To this gradiometer, we added within a distance of $35 \mathrm{~cm}$ a three-axis fluxgate magnetometer [15] as a reference system. This complete system was operated in three different environments and the performance and environmental noise rejection are compared.

TABLE II

Data of the different magnetometers used in the second order gradiometer configuration.

$\begin{array}{lccc} & \begin{array}{c}\mathrm{L}_{\mathrm{p}}[\mathrm{pH}] \\ \text { SQUID A }\end{array} & \begin{array}{c}\mathrm{A}_{\text {eff }} \\ {\left[\mathrm{mm}^{2}\right]}\end{array} & \begin{array}{c}\text { Typical white noise } \\ {[\mathrm{fT} / \mathrm{VHz}]}\end{array} \\ \text { SQUTD B } & 300 & 2.91 & 85 \\ \text { SQUID C } & 300 & 3.24 & 54 \\ & 300 & 3.18 & 49\end{array}$

Starting with the performance inside the shielded room we measured a white noise performance for the second order gradiometer of $120 \mathrm{fT} / \sqrt{\mathrm{Hz}}$ (Fig. 5). In comparison, the white noise level for the best magnetometer was $49 \mathrm{fT} / \sqrt{ } \mathrm{Hz}$. In the shielded room, this higher noise level for the second order gradiometer, together with the spatial filtering, results in a lower signal to noise ratio of the MCG recordings (Fig. 6). The line frequency peaks seen in the magnetometer spectrum in Fig. 5 are stronger in the gradiometer signal and are caused by the electronics. Despite the higher noise level in

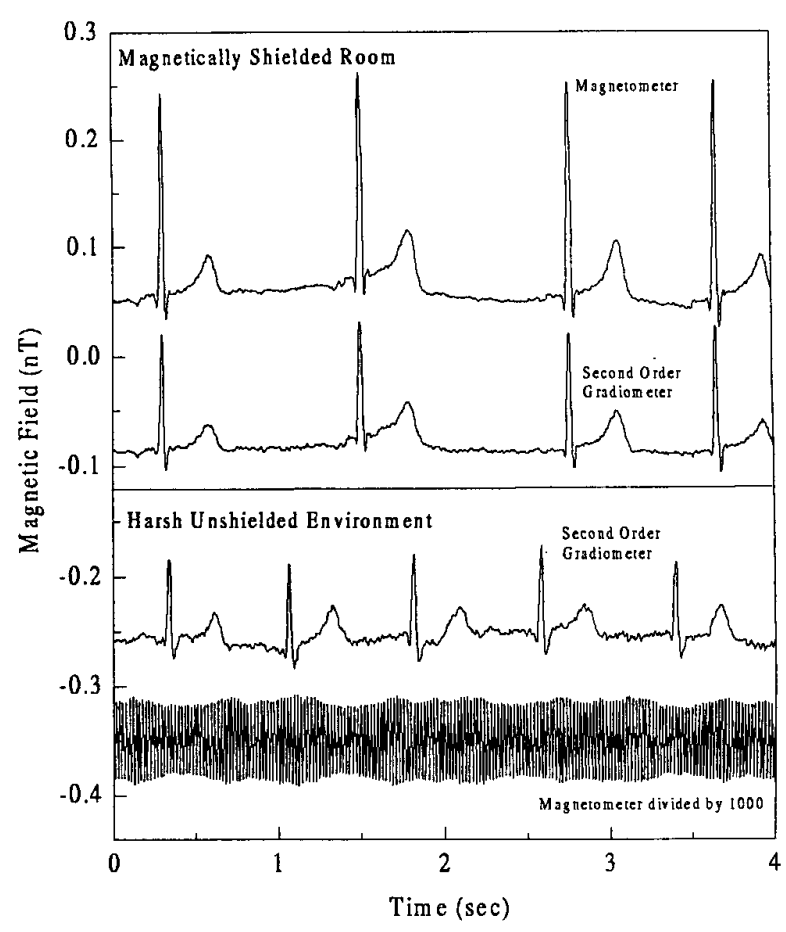

Fig.6. Time traces of magnetocardiograms for different adult persons measured with a second order gradiometer and magnetometer in magnetically shielded (MSR, Univ. of Twente) and harsh unshielded environment (EL/TN building, Univ. of Twente). The gradiometer signals and the shielded magnetometer are within a bandwidth of 0.1 to $47 \mathrm{~Hz}$

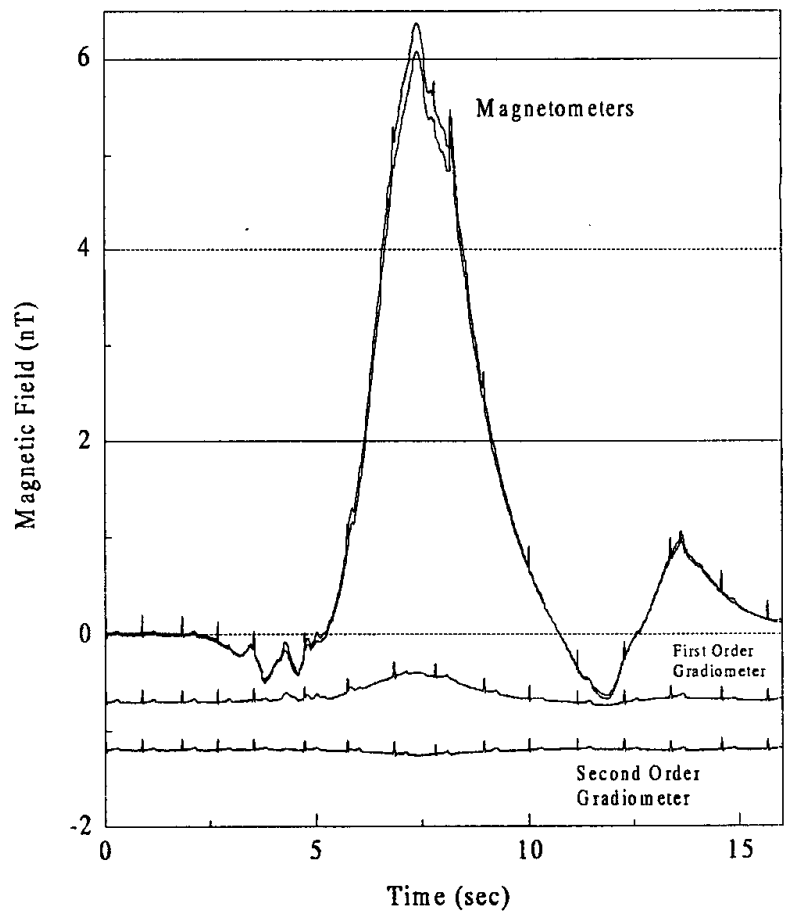

Fig.7. Simultaneously recorded time traces of the magnetometers during an MCG measurement in the shielded room in Twente in comparison to firstand second order gradiometer signals. The strong disturbance is caused by the closure of the door. The signals were bandpass filtered within 0.1 to $47 \mathrm{~Hz}$. 


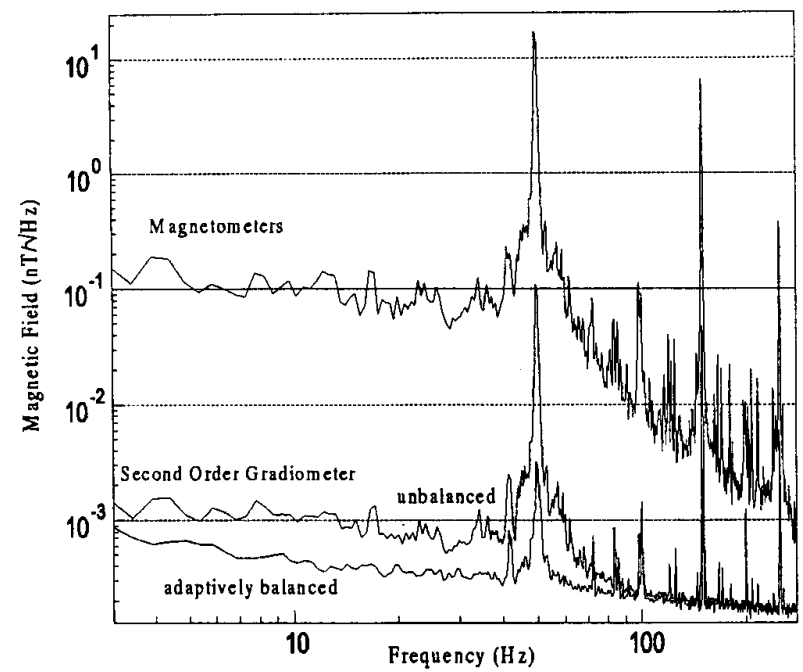

Fig.8. Simultaneously recorded spectral magnetic noise contributions of the SQUID magnetometers in comparison to the unbalanced and adaptively balanced second order gradiometer in harsh magnetically unshielded environment at EL TN building, University of Twente.

the second order gradiometer signal, the spatial filtering allows the efficient rejection of strong environmental disturbances during measurements inside shielded environments (Fig. 7).

In an unshielded environment with low disturbance, like the Biomagnetic Center Twente (BCT), the spectral magnetic noise distribution of the gradiometer is nearly the same as inside the shielded room. Only the $50 \mathrm{~Hz}$ line frequency and harmonics show an increase by a factor of 20 . By adding the signals of the reference system to the gradiometer, the line frequency interference peaks were suppressed by a factor of 10 , but the overall noise performance remained the same.

In unshielded environments with strong disturbances, like the EL/TN building of the University of Twente, the spectral noise is strongly increased in the magnetometers and the unbalanced second order gradiometer (Fig. 8). For this environment the application of the reference magnetometers showed strong improvements. As a first step we added the reference signal and the output of the two lower SQUID magnetometers to the second order gradiometer signal with fixed coefficients in an averaged adaptive process [11]. Without any correction in frequency for the different signals we achieved averaged common mode rejections up to $76 \mathrm{~dB}$ for the $50 \mathrm{~Hz}, 66 \mathrm{~dB}$ for the $150 \mathrm{~Hz}$ disturbances and $55 \mathrm{~dB}$ at lower frequencies. The use of the same coefficients on measurements taken 2 and 4 hours later resulted in the same noise level.

As a next step we plan to apply more sophisticated signal processing methods like frequency dependent adaptive methods [11] and filtered application of the reference signals. This should lead to a further improvement of the environmental noise rejection in harsh environment down to the intrinsic noise level of the gradiometer.

\section{CONCLUSION}

We have successfully tested two different types of gradiometer formation for the use in a harsh magnetically unshielded environment, fully based on HTS SQUID magnetometers. With the first technique we were able to suppress the signal caused by the movement in the earth's magnetic field by a factor of 50 without changing the general performance of the first order gradiometer. MCG measurements in an unshielded environment showed no difference in the signal with and without active compensation. Here, the active compensation allowed the system to be moved in a magnetically unshielded environment.

With the second technique we were able to demonstrate high common mode rejections of environmental noise. By adding the signals of the reference system to the signal of a second order electronic gradiometer with a white noise level of $120 \mathrm{fT} / \sqrt{\mathrm{Hz}}$, we were able to suppress the environmental noise near to the intrinsic noise of the gradiometer system. The power line disturbance at $50 \mathrm{~Hz}$ was suppressed by an average factor of 6700 . Further tests with frequency dependent coefficients should lead to even higher noise rejection values.

\section{ACKNOWLEDGMENT}

The authors would like to thank K. Barthel, J. Schubert and $W$. Zander at research center Jülich, Germany for providing us in parts with HTS rf-SQUIDs.

\section{REFERENCES}

[1] R. E. Sarwinski, Cryogenics 17, pp.671, 1977

[2] Y. Zhang, H. Soltner, H.J Krause, E. Sodtke, W. Zander, J. Schubert, M. Gruneklee, D. Lomparski, M. Banzet, H. Bousack, A.I. Braginski, IEEE Trans. On Appl. Supercond. 7, pp. 2866, 1997

[3] M.N. Keene, N.J. Exon, R.G. Humphreys, N.G. Chew, Journ. Appl. Phys. 79, pp. 8783, 1996

[4] E. Dantsker, O.M. Froehlich, S. Tanaka, K. Kouznetsov, and J. Clarke, Appl. Phys. Lett. 71, pp. 1712, 1997

[5] Y. Tavrin, Y. Zhang, M. Mück, A.I. Braginki, and C. Heiden, Appl. Phys. Lett. 62, pp. 1824, 1993

[6] Y. Tavrin, Y. Zhang, W. Wolf, and A. I. Braginski, Supercond. Sci. Technol.7,pp. 265, 1994

[7] J. Borgmann, G. Ockenfuss, R. Otto, J. Schubert, W. Zander, A. I. Braginski, and P. David, Rev. Sci. Instrum. 68, pp. 2730, 1997

[8] J. Borgmann, P. David, H.J. Krause, R. Otto, and A. I. Braginski, Rev. Sci. Instrum. 68, pp. 3082, 1997

[9] R. H. Koch, J. R. Rozen, J. Z. Sun, and W. J. Gallagher, Appl. Phys. Lett. 63, pp. 3, 1993

[10] H.J.M. ter Brake, Z. Dunajski, W.A.G. van der Mheen and J. Flokstra, J. Phys. E: Sci. Instrum. 22, pp. 560, 1989

[11] J. Vrba, in "SQUID Sensors: Fundamentals, Fabrication and Applications", ed. H. Weinstock, Kluwer Academic Publishers, Dordrecht, pp.117, 1996

[12] M.J. Peters, University of Twente, Netherlands, personal communication, 1998

[13] A.P. Rijpma, J. Borgmann, H.J.M ter Brake and H. Rogalla Appl. Supercond Conf., Palm Springs, California 1998

[14] Y. Zhang, H.-M. Mück, K. Hermann, J. Schubert, W. Zander, A.I. Braginski, Appl. Phys. Lett. 60, pp. 645, 1992

[15] Bartington Instr. Ltd, Oxford, England, Operation manual OX8 7GE, 1996 